\title{
ИМИТАЦИОННОЕ МОДЕЛИРОВАНИЕ ПОЛУМАРКОВСКИХ ПРОЦЕССОВ В СИСТЕМАХ С ДИСКРЕТНЫМИ СОСТОЯНИЯМИ И НЕПРЕРЫВНЫМ ВРЕМЕНЕМ
}

\author{
Л. Б. Афанасьевский ${ }^{\star}$ А. Н. Горин ${ }^{\star}$ М. А. Чурсин ${ }^{*}$ \\ ${ }^{*}$ ВУНЦ ВВС «Военно-воздушная академия им. проф. Н. Е. Жуковского и Ю. А. Гагарина» (2. Воронеж) \\ ** Воронежский филиал Российского экономического университета им. Г.В. Плеханова
}

Поступила в редакцию 10.07.2019 г.

\begin{abstract}
Аннотация. Решена задача построения имитационной модели функционирования сложных систем, построенных по блочно-модульному принципу, в которых протекают двумерные полумарковские процессы. Эти процессы определяют последовательность смены дискретных состояний, характеризующих частичные или полные потери функциональных возможностей систем. Процесс, изменяющий состояния, является вложенным по отношению к процессу, формирующему время нахождения системы в состояниях. Потоки событий, обеспечивающих смену состояний и задающих время пребывания системы в состояниях, имеют произвольные распределения вероятностей. Результатом моделирования являются оценки стационарных вероятностей состояний. Повышение точности оценок вероятностей обеспечивается с помощью их рекуррентного усреднения по нарастающему количеству реализаций модели. Это позволяет использовать имитационные модели для определения характеристик систем при изменении их параметров. Корректность имитационной модели подтверждена сравнением результатов имитационного моделирования с результатами расчетов по аналитическим формулам. На примере показано, что имитационная модель позволяет получить результаты с той же точностью значительно проще, чем с помощью расчетов по аналитическим формулам. Возможные пути использования оценок вероятностей - помощь в принятии решений о соответствии систем предъявляемым к ним требованиям, проверка корректности аналитических моделей.

Ключевые слова: имитационная модель, полумарковский процесс, стационарные вероятности состояний.
\end{abstract}

\section{ВВЕДЕНИЕ}

При разработке и исследовании сложных систем с дискретными состояниями достаточно широко используются аналитические полумарковские модели их функционирования, например $[1,2]$, при этом одним из результатов моделирования являются оценки вероятностей нахождения системы в различных состояниях (вероятностей состояний) в установившемся режиме.

Определение стационарных вероятностей состояний для полумарковских процессов представляет собой достаточно сложную вычислительную задачу [3-6], сводящуюся к многоэтапным расчетам. Кроме того, сами 2019

(c) Афанасьевский Л. Б., Горин А. Н., Чурсин М. А., результаты могут содержать погрешности как из-за сложностей моделей, так и применения численных методов для получения оценок вероятностей.

Подтвердить результаты, полученные с использованием аналитических моделей, можно с помощью имитационных моделей [7], позволяющих учесть нюансы функционирования систем. Имитационное моделирование может быть использовано и как основной инструментарий при исследовании сложных систем $[8,9]$.

Следует отметить, что при имитационном моделировании широко используется вычислительная система Matlab [2, 7].

Для каждого сеанса имитационного моделирования используется одна точка в пространстве параметров исследуемой системы. Влияние параметров системы на вероятности 
состояний устанавливается путем построения и реализации плана вычислительных экспериментов на модели в области определения параметров.

\section{1. ПОСТАНОВКА ЗАДАЧИ}

В ходе подготовки специалистов по направлению «Специальные радиотехнические системы» в ВУНЦ ВВС в дисциплине «Основы компьютерного проектирования и моделирования радиотехнических систем» ставится задача разработки имитационных моделей сложных систем с дискретными состояниями для случая, когда случайные процессы, протекающие в системе, являются полумарковскими. Разработка такой модели является существенным элементом формирования у обучающихся навыков экспериментальных исследований, анализа полученных результатов, оценки их достоверности и адекватности.

Результатом работы модели должны быть оценки стационарных вероятностей состояний системы.

В основу построения моделей положены следующие положения, которые позволяют считать системы полумарковскими:

1) изменение состояний систем происходит случайным образом, т. е. системы являются стохастическими;

2) изменение состояний и времени нахождения системы в состояниях после переходов осуществляются двумя независимыми множествами потоков с произвольными распределениями вероятностей;

3) статистические свойства процессов, изменяющих состояния и определяющих время пребывания системы в состояниях, не зависят от числа уже сделанных переходов (системы являются однородными) и от способа попадания системы в каждое из возможных состояний;

4) изменение состояний системы происходит мгновенно.

При разработке моделей должна быть реализована возможность уменьшения разброса вероятностей состояний, обусловленного стохастическим характером имитационной модели, до практически приемлемого уровня путем использования современных методов обработки измерений.

На этапе разработки моделей должна быть подтверждена их правильность путем сравнения результатов с результатами, полученными другими методами.

\section{2. АНАЛИТИЧЕСКИЙ МЕТОД РАСЧЕТА ПОЛУМАРКОВСКОЙ СИСТЕМЫ}

Имитационную модель будем считать корректной, если оценки вероятностей, полученные на имитационной модели, с приемлемой точностью будут совпадать с оценками, полученными аналитическим методом.

В рассматриваемой постановке задачи полумарковский процесс является двумерным. Процесс, изменяющий состояния, является вложенным по отношению к процессу, формирующему время нахождения системы в состояниях.

Для двумерного процесса процедура определения стационарных вероятностей состояний состоит в проведении многоэтапных расчетов.

Стационарные вероятности полумарковского процесса определяются по формуле [3]

$$
p_{j}=\frac{a_{j} m_{j}}{\sum_{i=1}^{n} a_{i} m_{i}}, j=1,2, \ldots, n,
$$

где $a_{j}, j=1,2, \ldots, n$ - стационарные вероятности состояний вложенного процесса;

$m_{i}, i=1,2, \ldots, n$ - среднее время пребывания в $i$-м состоянии при произвольном распределении вероятностей.

Среднее время пребывания процесса в $i$-м состоянии определяется по формуле

$$
m_{i}=\sum_{k=1}^{n} q_{i k} \int_{0}^{\infty} t f_{i k}(t) d t,
$$

где $n$ - число состояний; $f_{i j}(t)$ - плотность вероятности для случайного времени пребывания процесса в $i$-м состоянии, если следующий переход будет в состояние $j ; q_{i j}$, $i, j=1,2, \ldots, n$ - переходные вероятности в стационарном режиме, вычисляемые по формуле [6] 


$$
q_{i j}=\int_{0}^{\infty} \prod_{\substack{k=1 \\ k \neq i}}^{n}\left(1-F_{i k}(t)\right) d F_{i j}(t), i, j=1,2, \ldots, n .
$$

В формуле (3) $F_{i j}(t), \quad i, j=1,2, \ldots, n-$ функции распределения для потоков событий, определяющих время пребывания системы в $i$-м состоянии, если следующий переход будет выполнен в состояние $j$.

Стационарные вероятности для вложенного процесса вычисляются из системы линейных уравнений

$$
a_{i}=\sum_{j=1}^{n} a_{j} q_{j i}, i=1,2, \ldots, n,
$$

в которой одно из уравнений заменяется условием нормировки

$$
\sum_{i=1}^{n} a_{i}=1
$$

Приведенные формулы (1)-(4) позволяют сделать следующие выводы:

оценки стационарных вероятностей можно получить только численными методами;

в расчетных соотношениях в явном виде должны использоваться формулы для плотностей вероятностей и функций распределения;

расчетные соотношения неудобны для программирования;

сложно получить зависимости вероятностей состояний от параметров распределений.

Эти сложности в значительной степени исчезают при использовании имитационных моделей.

\section{3. ИМИТАЦИОННОЕ МОДЕЛИРОВАНИЕ ДВУМЕРНЫХ ПОЛУМАРКОВСКИХ ПРОЦЕССОВ}

Имитационная модель, по сути, представляет собой самостоятельный объект (систему), на котором проводятся статистические испытания. Каждое испытание представляет собой одну реализацию модели при некоторых значениях параметров. В результате испытания система переходит в некоторое другое состояние и остается в нем в течение случайного времени.

Проведя достаточно большое число испытаний $N$ и подсчитав суммарное время Tsost $_{i}, i=1,2, \ldots, n$ нахождения системы в со- стоянии $S_{i}$ и общее время нахождения во всех состояниях $T s$, искомые вероятности можно определить по формуле

$$
P_{i}=\frac{T_{\text {Sost }}}{T s} .
$$

Естественно, имитационная модель должна объединять в себе наиболее существенные свойства реального объекта.

Построим имитационную модель функционирования систем, в которых состояния изменяются мгновенно, при этом моменты смены состояний задаются непрерывными функциями времени (плотностями вероятностей), вид и параметры которых зависят от направлений переходов из состояний. Кроме того, система после перехода в некоторое состояние остается в нем в течение некоторого случайного времени, также зависящего от направления перехода. Разработку будем вести в среде Matlab, вид плотностей вероятностей и функций распределения будет ориентирован на эту среду.

Модель такой системы представляет собой двумерный полумарковский процесс [3, $4,6]$. В этом двумерном процессе переход в новое состояние осуществляется вложенным полумарковским процессом.

Алгоритм имитационной модели включает в себя следующие операции:

определение номера $k$ исходного состояния по известным начальным вероятностям состояний $P_{i}(0), i=1,2, \ldots, n$;

организацию большого числа $N$ испытаний модели, при этом для каждого испытания определяется, в какое состояние должна перейти система.

После завершения $N$ испытаний вычисляются оценки стационарных вероятностей в соответствии с формулой (5).

Номер начального состояния $k$ определяется путем «розыгрыша» кодом, представленным в листинге 1.

Процедура выбора состояния, в которое должна перейти система из текущего состояния, следующая:

генерируются случайные числа в соответствии с плотностями вероятностей для всех потоков, переводящих систему из текущего состояния в другие возможные состояния; 
$\mathrm{s}=\mathrm{p}(1) ; \mathrm{k}=1 ;$

gg=rand; оПолучение случайного числа, равномерно ораспределенного в $[0 ; 1]$

while $(g \mathrm{~g}>\mathrm{s}), \mathrm{k}=\mathrm{k}+1 ; \mathrm{s}=\mathrm{s}+\mathrm{p}(\mathrm{k})$;

где $p$ - одномерный массив начальных вероятностей состояний.

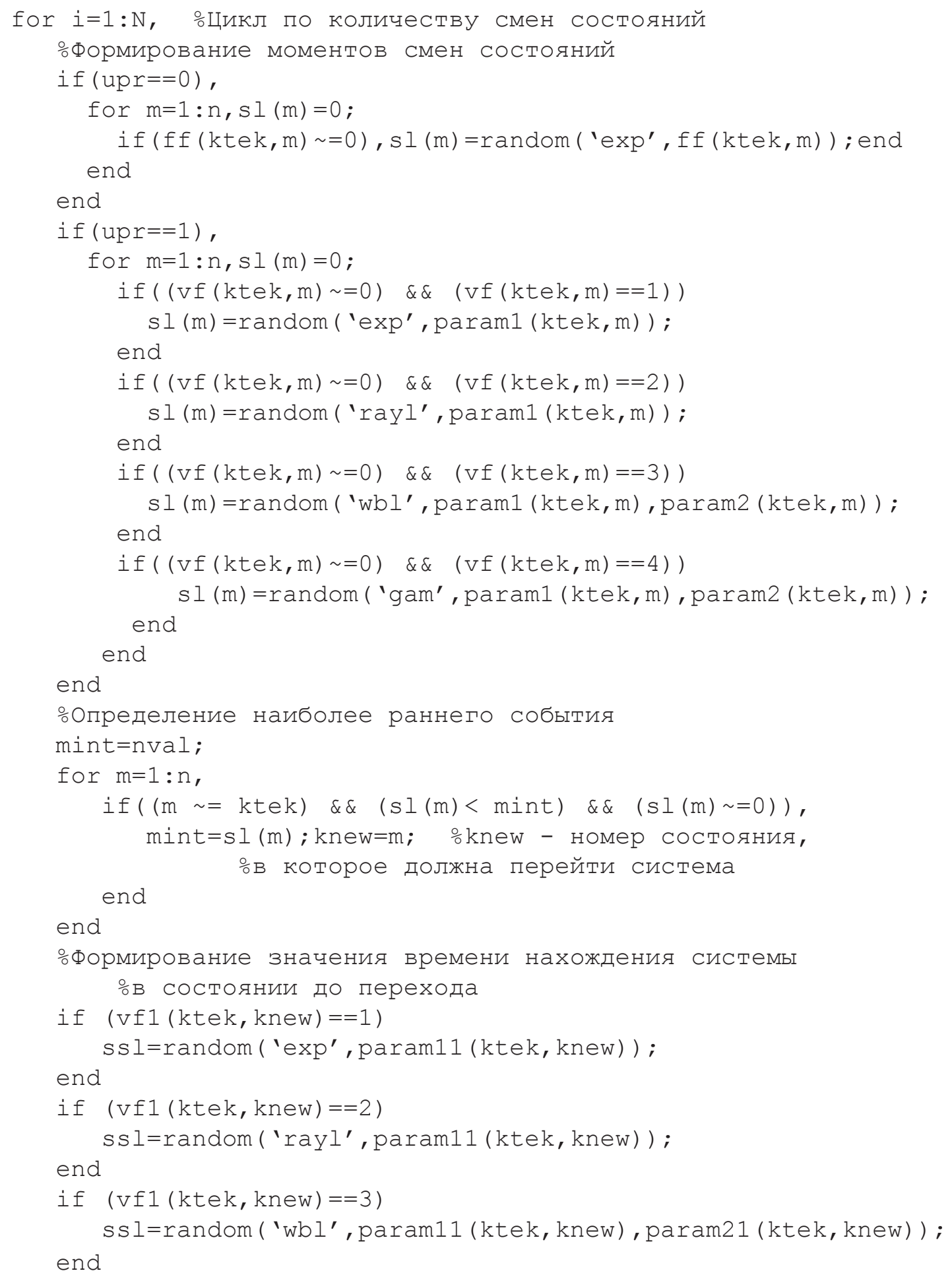


Листинг 2 (продолжение)

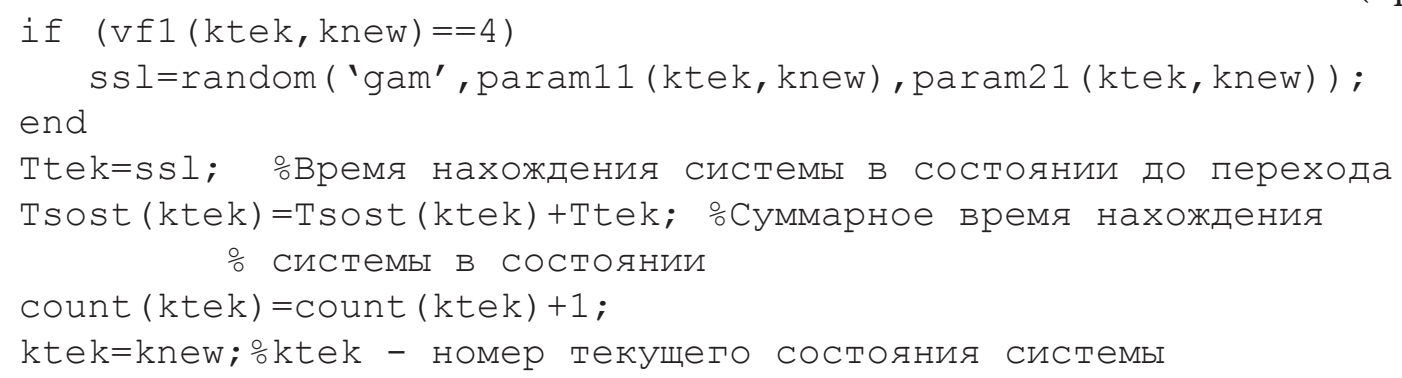

выбирается минимальное случайное число, соответствующее наступлению ближайшего события, и фиксируется номер состояния, в которое должна перейти система.

Далее в зависимости от того, в какое состояние должна перейти система, формируется с учетом соответствующей плотности вероятности случайное значение времени нахождения системы в текущем состоянии.

Основная часть имитационной модели на языке Matlab представлена в листинге 2.

Управляющий параметр upr задает режим работы модели. При $u p r=0$ смена состояний осуществляется непрерывной марковской цепью, при $u p r=1$ - полумарковским потоком событий.

В приведенном коде имитационной модели предусмотрено применение четырех наиболее часто используемых распределений: экспоненциального (exp - код 1), Рэлея (rayl - код 2), Вейбулла ( $w b l$ - код 3), Гамма-распределения ( gam - код 4).

Естественно, что количество видов распределений может быть легко увеличено путем добавления в код соответствующих условных операторов.

В качестве исходных данных для такой модели должны использоваться шесть матриц, если ограничиться двухпараметрическими плотностями вероятностей.

В матрице $v f$ указываются коды вида плотностей вероятностей для каждого варианта перехода из состояния, а в матрицах param1 и param2 - параметры плотностей вероятностей.

В матрице $v f 1$ указываются коды вида плотностей вероятностей для времен нахождения системы в состояниях, а в матрицах param11 и param21 - параметры плотностей вероятностей.

Генерирование случайных чисел для различных законов распределения в Matlab ocyществляется с помощью функции random. Обращение к этой функции имеет вид

$$
x=\operatorname{random}(\text { 'name', valuel[ }[\text { value } 2, \ldots]),
$$

где $x$ - случайное число; name - название закона распределения, принятое в Matlab; value1, value 2 - значения параметров плотности вероятности.

Оценки вероятностей состояний $P_{i}$, $i=1,2, \ldots, n$ в установившемся режиме вычисляются как отношения времени нахождения системы в конкретных состояниях ко времени нахождения системы во всех состояниях при достаточно большом количестве испытаний модели.

\section{4. ВИЗУАЛИЗАЦИЯ РЕЗУЛЬТАТОВ МОДЕЛИРОВАНИЯ И ПОВЫШЕНИЕ ТОЧНОСТИ ОЦЕНОК}

B Matlab имеются различные средства визуализации результатов расчетов. Исследователь может использовать возможности визуализации для принятия решения о необходимом числе испытаний имитационной модели, необходимости применения каких-либо методов для обработки результатов моделирования.

В имитационной модели можно достаточно легко дополнительно организовать цикл по количеству испытаний (переходов) $N$ и сохранить результаты в двумерном массиве $x x$.

В приведенном коде (листинг 3) массив $x$ содержит значения стационарных вероятностей для текущего числа испытаний модели $N$. 


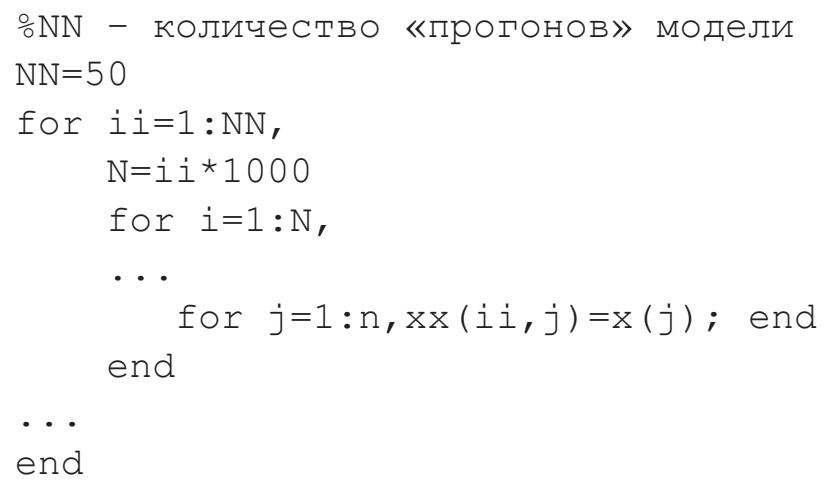

График зависимостей вероятностей от числа испытаний модели, например для четырех состояний, может быть сформирован кодом, приведенным в листинге 4.

Зависимости вероятностей состояний от количества переходов (шаг равен 1000) для параметров, указанных в матрицах (листинг 5) приведены на рис. 1.

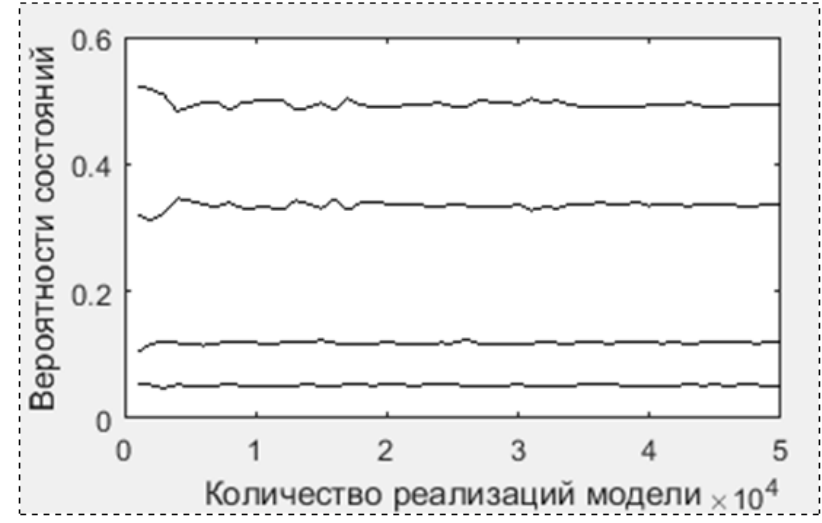

Рис. 1. Зависимости вероятностей состояний от количества переходов

В имитационной модели эмпирические значения вероятностей определяются по формуле (5). Естественно, оценки вероятно- стей могут считаться относительно достоверными только при больших значениях $N$. Но даже при больших значениях $N$ будут наблюдаться флуктуации в значениях вероятностей при повторных «прогонах» модели изза ее стохастического характера (рис. 1).

Сглаживание «зашумленных» измерений может выполняться различными методами, например усреднением оценок вероятностей, полученных по множеству «прогонов» модели при одинаковых количествах переходов между состояниями.

В рассматриваемом случае модель системы представляет собой самостоятельный объект, на котором проводятся статистические эксперименты. Результаты этих экспериментов, полученные на модели при разных значениях переходов $N$, могут быть использованы для уточнения оценок вероятностей с помощью рекуррентного определения математического ожидания для вероятности $j$-го состояния.

$$
\begin{gathered}
m_{j}(k)=m_{j}(k-1)+\frac{1}{k}\left[x_{j}(k)-m_{j}(k-1)\right], \\
k=1,2, \ldots, N N,
\end{gathered}
$$


где $N N$ - количество «прогонов» модели;

$m_{j}$ - среднее значение параметра $x_{j}$;

$x_{j}(k)$ - значение параметра $x_{j}$ при $k$-м сеансе моделирования.

Зависимости вероятностей состояний от количества переходов при сглаживании вероятностей с помощью рекуррентного усреднения (6) приведены на рис. 2.

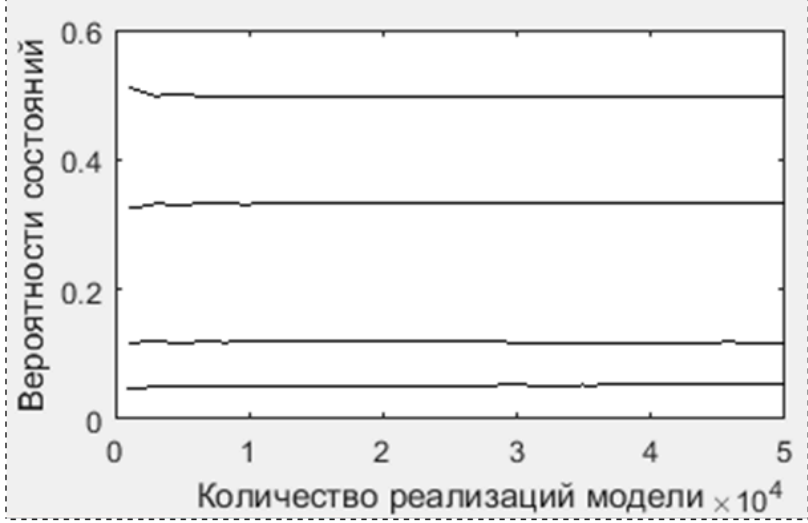

Рис. 2. Зависимости вероятностей состояний от количества переходов при рекуррентном усреднении

Результаты, представленные на рис. 2, получены при тех же исходных данных, что и для рис. 1.

\section{5. ПРИМЕР ИСПОЛЬЗОВАНИЯ АНАЛИТИЧЕСКОЙ И ИМИТАЦИОННОЙ МОДЕЛЕЙ}

В качестве примера системы, описываемой двумерной полумарковской моделью, рассмотрим специальное радиоэлектронное средство (РЭС), поведение которого задано графом, представленным на рис. 3 [10].

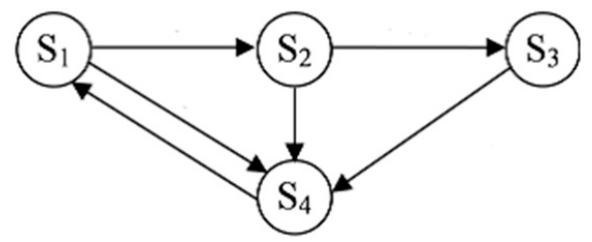

Рис. 3. Граф состояний и переходов РЭС

На рис. 3 приняты следующие обозначения:

$S_{1}$ - РЭС находится в боевой готовности; $S_{2}$ - РЭС ведет разведку целей; $S_{3}$ - РЭС ведет подавление целей; $S_{4}-$ РЭС находится в неисправном состоянии.

Примем, что поведение такой системы описывается двумерной полумарковской моделью.

Оценим вероятности состояний в стационарном режиме для исходных данных, приведенных в разд. 4.

Для подтверждения правильности имитационной модели сравним результаты расчетов по формулам (1)-(4) с результатами имитационного моделирования полумарковского процесса для системы, граф которой изображен на рис. 3.

Исходные данные для моделирования приведены в табл. 1-2.

Среднее значение $m_{x}$ для распределения Рэлея вычисляется по формуле $m_{x}=b \sqrt{\frac{\pi}{2}}$, а для распределения Вейбулла - по формуле $m_{x}=a \Gamma(1+1 / b)$, где $\Gamma(\cdot)$ - гамма-функция, $a$ - параметр масштабирования, $b$ - параметр формы.

Стационарные вероятности состояний (psostimits), полученные с помощью имитационной модели, равны

Таблица 1

Виды и параметры плотностей вероятностей для времени между переходами

\begin{tabular}{|c|c|c|c|}
\hline $\begin{array}{c}\text { Переход между } \\
\text { состояниями }\end{array}$ & $\begin{array}{c}\text { Вид и код } \\
\text { распределения }\end{array}$ & $\begin{array}{c}\text { Параметры } \\
\text { распределения }\end{array}$ & $\begin{array}{c}\text { Среднее значение } \\
\text { времени между } \\
\text { сменами состояний }\end{array}$ \\
\hline $1-2$ & Рэлея -2 & $b=5$ & 6,27 \\
\hline $1-4$ & Рэлея -2 & $b=6$ & 7,52 \\
\hline $2-3$ & Вейбулла -3 & $a=2,8 ; b=2$ & 2,48 \\
\hline $2-4$ & Экспоненциальное -1 & $m=2,5$ & 2,5 \\
\hline $3-4$ & Экспоненциальное -1 & $m=2,5$ & 2,5 \\
\hline $4-1$ & Экспоненциальное -1 & $m=1,4$ & 1,4 \\
\hline
\end{tabular}


Таблица 2

Виды и параметры плотностей вероятностей для времени нахождения системы в состояниях

\begin{tabular}{|c|c|c|c|}
\hline $\begin{array}{c}\text { Переход между } \\
\text { состояниями }\end{array}$ & $\begin{array}{c}\text { Вид и код } \\
\text { распределения }\end{array}$ & $\begin{array}{c}\text { Параметры } \\
\text { распределения }\end{array}$ & $\begin{array}{c}\text { Среднее значение } \\
\text { времени нахождения } \\
\text { системы в состояниях }\end{array}$ \\
\hline $1-2$ & Экспоненциальное -1 & $m=4$ & 4 \\
\hline $1-4$ & Экспоненциальное -1 & $m=3,9$ & 3,9 \\
\hline $2-3$ & Рэлея - & $b=1,5$ & 1,88 \\
\hline $2-4$ & Рэлея -2 & $b=12,5$ & 2,5 \\
\hline $3-4$ & Экспоненциальное -1 & $m=2,5$ & 1,4 \\
\hline $4-1$ & Экспоненциальное - 1 & $m=1,4$ & \\
\hline
\end{tabular}

Таблица 3

Результаты расчетов по аналитическим формулам

\begin{tabular}{|c|llll|c|c|}
\hline $\begin{array}{c}\text { Среднее время } m_{i} \\
\text { пребывания } \\
\text { в состоянии }\end{array}$ & \multicolumn{2}{|l|}{ Переходные вероятности $q_{i j}$} & $\begin{array}{c}\text { Стационарные } \\
\text { вероятности } \\
\text { для вложенного } \\
\text { процесса }-a_{i}\end{array}$ & $\begin{array}{c}\text { Стационарные } \\
\text { вероятности } \\
\text { состояний } p_{i}\end{array}$ \\
\hline$m_{1}=3,959$ & 0 & 0,59 & 0 & 0,41 & 0,353 & 0,3352 \\
\hline$m_{2}=9,904$ & 0 & 0 & 0,418 & 0,582 & 0,208 & 0,4941 \\
\hline$m_{3}=2,5$ & 0 & 0 & 0 & 1 & 0,087 & 0,0522 \\
\hline$m_{4}=1,4$ & 1 & 0 & 0 & 0 & 0,353 & 0,1185 \\
\hline
\end{tabular}

$$
N N=50
$$

psostmods $=\left[\begin{array}{llll}0.336 & 0.4929 & 0.0528 & 0.1183\end{array}\right]$ где $N N$ - число сеансов моделирования с нарастающим количеством переходов при шаге, равном 1000: (1000:1000: $\left.N N^{\star} 1000\right)$.

Результаты расчетов по формулам (1)-(4) приведены в табл. 3 и с точностью до второго знака после запятой совпадают с результами имитационного моделирования.

Близость значений стационарных вероятностей для обоих видов моделей подтверждает корректность подхода при построении имитационной модели.

Результаты, приведенные в табл. 3, получены в Mathcad. Ниже в качестве примера приведены фрагменты расчетов.

$$
\begin{gathered}
a 12:=5 \quad \text { a14 }:=6 \\
P 12:=\int_{0}^{\infty} e^{\frac{-x^{2}}{2 \cdot a 14^{2}}}\left[\frac{d}{d x}\left(1-e^{\frac{-x^{2}}{2 \cdot a 12^{2}}}\right)\right] d x=0.59 \\
b 23:=1.5 \quad b 24:=12.5
\end{gathered}
$$

$$
\begin{aligned}
& m 2:=0.418 \cdot \int_{0}^{\infty} \frac{t^{2}}{b 23^{2}} e^{\frac{-t^{2}}{2 \cdot b 23^{2}}} d t+ \\
& +0.582 \cdot \int_{0}^{\infty} \frac{t^{2}}{b 24^{2}} e^{\frac{-t^{2}}{2 \cdot b 24^{2}}} d t=9.904 \\
& A:=\left(\begin{array}{cccc}
1 & 0 & 0 & -1 \\
-0.59 & 1 & 0 & 0 \\
0 & -0.418 & 1 & 0 \\
1 & 1 & 1 & 1
\end{array}\right) \\
& B:=\left(\begin{array}{l}
0 \\
0 \\
0 \\
1
\end{array}\right) \quad a:=A^{-1} \cdot B=\left(\begin{array}{l}
0.353 \\
0.208 \\
0.087 \\
0.353
\end{array}\right) \\
& n:=4 \quad m:=\left(\begin{array}{llll}
3.959 & 9.904 & 2.5 & 1.4
\end{array}\right)^{T} \\
& a:=\left(\begin{array}{llll}
0.353 & 0.208 & 0.087 & 0.353
\end{array}\right)^{T}
\end{aligned}
$$




$$
i:=1 . . n \quad p_{i}:=a_{i} \cdot \frac{m_{i}}{\sum_{j=1}^{n}\left(m_{j} \cdot a_{j}\right)} \quad p=\left(\begin{array}{l}
0.3352 \\
0.4951 \\
0.0522 \\
0.1185
\end{array}\right)
$$

Аналитические методы определения стационарных вероятностей полумарковских процессов достаточно сложны, поэтому использование имитационных моделей в качестве основного рабочего инструмента в этом случае является оправданным.

\section{6. ОПРЕДЕЛЕНИЕ ХАРАКТЕРИСТИК СИСТЕМЫ НА МОДЕЛИ}

Из рис. 2 следует, что оценки вероятностей, по сути, являются «точечными», следовательно, имитационные модели можно использовать и для исследования характеристик системы при изменениях ее параметров.

На практике каждое состояние обычно характеризуется некоторым набором параметров. Эти параметры тем или иным образом влияют на параметры плотности вероятности, определяющей либо процесс смены состояний, либо значение времени нахождения системы в состоянии.

В качестве примера на рис. 4 приведены зависимости вероятностей состояний от параметра масштабирования распределения Рэлея для времени нахождения системы в состоянии 2 при переходе в состояние 4 [значения элемента param $11(2,4)]$.

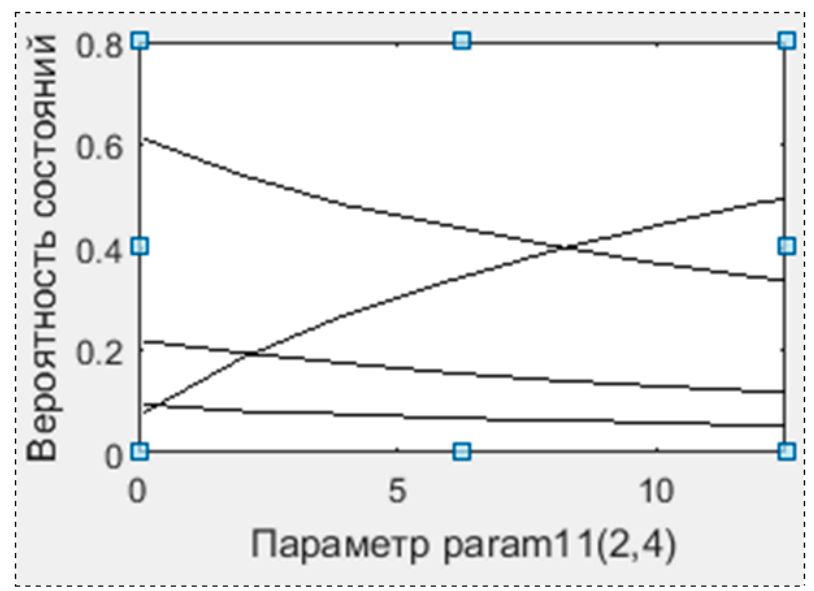

Рис. 4. Зависимости вероятностей состояний от параметра масштабирования распределения Рэлея

\section{ЗАКЛЮЧЕНИЕ}

Таким образом, разработана имитационная модель, позволяющая:

упростить подготовку исходных данных для определения стационарных вероятностей состояний по сравнению с аналитическими моделями;

получить «точечные» оценки вероятностей состояний при использовании рекуррентного усреднения;

исследовать влияние параметров на значения вероятностей состояний;

легко получить значения количеств переходов в каждое из состояний и средние значения времени нахождения в состояниях;

протоколировать процесс смены состояний и визуализировать результаты моделирования за счет возможностей, предоставляемых Matlab.

Кроме того, положительной стороной имитационной модели является ее универсальность. Она может быть легко настроена на исследование систем с любым числом состояний и потоков событий, имеющих произвольные распределения.

Разработанная модель может использоваться как для проведения практических расчетов, так и для проверки корректности аналитических методов расчета, она внедрена в учебный процесс ВУНЦ ВВС и позволяет готовить обучающихся к научно-исследовательской деятельности.

В ходе разработки и исследовании модели у обучающихся расширяется кругозор знаний по пакетам прикладных программ для моделирования сложных систем, по методам исследования и разработки алгоритмов реализации математических моделей объектов и процессов.

Будущие военные специалисты обеспечиваются знаниями по основам аналитического и имитационного моделирования объектов и процессов применения и функционирования сложных систем, у них формируются умения в применении компьютерных систем и пакетов прикладных программ для моделирования объектов и процессов с целью анализа и 
оптимизации их параметров, выработка навыков в построении математических моделей объектов и процессов, выборе алгоритмов их компьютерной реализации.

\section{СПИСОК ЛИТЕРАТУРЫ}

1. Андреещев, И. А. Полумарковская модель оценки надежности функционирования автоматизированной системы управления силами и средствами радиоэлектронной борьбы / И. А. Андреещев, С. А. Будников // Вестник военно-воздушной академии. - 2019. № 2 (32). - С. 283-288.

2. Борисевич, А. В. Полумарковская модель для оценки показателей надежности источника бесперебойного питания дата-центра [Электронный ресурс] / А. В. Борисевич, Н. В. Дякин // Современные научные исследования и инновации. - 2015. - № 8. Ч. 1. - Режим доступа: http://web.snauka.ru/ issues/2015/08/57039. - (Дата обращения: 12.01.2018).

3. Андронов, А. М. Теория вероятностей и математическая статистика: учебник для вузов / А. М. Андронов, Е. А. Копытов. Л. Я. Гринглаз. - Санкт-Петербург: Питер, 2004. -461 c.

4. Тихонов, В. И. Марковские процессы / В. И. Тихонов, М. А. Миронов. - Москва : Советское радио, 1977. - 488 с.
5. Коваленко, И. Н. Полумарковские модели в задачах проектирования систем управления летательными аппаратами / И. Н. Коваленко, Г. К. Москатов, Е. Ю. Барзилович. Москва : Машиностроение, 1973. - 176 с.

6. Xie, W. Analysis of a two-level software rejuvenation polisy / W. Xie, Y. Hong, K. Trivedi // Reliability Engineering and System Safety, 2005. - V. 87. - P. 13-22.

7. Гультяев, А. К. Визуальное моделирование в среде Matlab. - Санкт-Петербург : Питер, 2000. - 432 c.

8. Афбанасьевский, Л. Б. Метод имитационного моделирования как средство анализа стохастических сетевых графиков / Л. Б. Афанасьевский, А. Н. Горин, М. А. Чурсин // Вестник Воронеж. гос. ун-та. Сер. Системный анализ и информационные технологии. - 2015. № 2. - C. 54-59.

9. Абанасьевский, Л. Б. Имитационное моделирование систем, управляемых событиями, в среде MatLab+Simulink / Л. Б. Афанасьевский, А. Н. Горин, А. Г. Фадин // Информатика: проблемы, методология, технологии : сб. тр. XVIII Междунар. науч.-метод. конф. Том 2 (Воронеж, 15-16 февраля 2018 г.). - Воронеж, Научно-исследовательские публикации, 2018. - С. 46-50.

10. Фадин, А. Г. Моделирование радиоэлектронных систем на ЭВМ: учебник / А. Г. Фадин.- Воронеж : ВИРЭ, 2000. - 493 с.

Афанасьевский Леонид Борисович - канд. техн. наук, доцент, доцент, ВУНЦ ВВС «Военно-воздушная академия им. проф. Н. Е. Жуковского и Ю. А. Гагарина» (г. Воронеж), e-mail: afleonid@yandex.ru

Горин Александр Николаевич - канд. техн. наук, ст. преподаватель, ВУНЦ ВВС «Военно-воздушная академия им. проф. Н.Е. Жуковского и Ю.А. Гагарина» (г. Воронеж), e-mail: algorin.algoral@mail.ru

Чурсин Михаил Александрович - канд. техн. наук, доцент, доцент, Воронежский филиал Российского экономического университета им. Г. В. Плеханова. 


\title{
IMITATION MODELING OF SEMI-MARKOV PROCESSES IN SYSTEMS WITH DISCRETE STATES AND CONTINUOUS TIME
}

\author{
L. B. Afanasievsky ${ }^{\star}$, A. N. Gorin ${ }^{\star}$, M. A. Chursin ${ }^{\star *}$ \\ ${ }^{*}$ Military Educational and Scientific Center of the Air Force «N. E. Zhukovsky and Y. A. Gagarin \\ Air Force Academy» (Voronezh), \\ **Voronezh branch of Russian economic University prof. G. V. Plekhanov
}

\begin{abstract}
Annotation. The problem of constructing a simulation model for the operation of complex systems, built on a block-modular principle, in which two-dimensional semi-Markov processes take place, has been solved. These processes determine the sequence of change of discrete states that characterize partial or complete loss of the functionality of the systems. The state changing process is nested with respect to the process that forms the time the system is in the states. The flows of events that provide for the change of states and determine the time the system is in the states have arbitrary probability distributions. The simulation results are estimates of stationary probabilities of states. Improving the accuracy of probability estimates is provided by their recurrent averaging over an increasing number of model realizations. This allows the use of simulation models to determine the characteristics of systems when their parameters change. The correctness of the simulation model is confirmed by comparing the results of simulation with the results of calculations using analytical formulas. The example shows that the simulation model allows you to get results with the same accuracy is much easier than using calculations using analytical formulas. Possible ways of using probability estimates are help in making decisions about the compliance of systems with their requirements, checking the correctness of analytical models.
\end{abstract}

Keywords: simulation model, semi-Markov process, stationary probabilities of states.

Afanasievsky Leonid Borisovitch - Candidate of technical sciences, Senior Lecturer, Military Educational and Scientific Center of the Air Force «N.E. Zhukovsky and Y.A. Gagarin Air Force Academy» (Voronezh), e-mail: afleonid@yandex.ru

Gorin Alexandr Nikolaevich - Candidate of technical sciences, Senior Lecturer, Military Educational and Scientific Center of the Air Force «N.E. Zhukovsky and Y.A. Gagarin Air Force Academy» (Voronezh), e-mail: algorin.algoral@mail.ru

Chursin Mikhail Aleksandrovitch - Candidate of Technical Sciences, Senior Lecturer, Voronezh branch of Russian State trade and economic University. 